\title{
Study on the Cross Plane Thermal Transport of Polycrystalline Molybdenum Nanofilms by Applying Picosecond Laser Transient Thermoreflectance Method
}

\author{
Tingting Miao, ${ }^{1,2}$ Weigang Ma, ${ }^{2,3}$ Xing Zhang, ${ }^{2,3}$ Keisuke Kubo, ${ }^{4}$ \\ Masamichi Kohno, ${ }^{3,4}$ Yasuyuki Takata, ${ }^{3,4}$ Tatsuya Ikuta, ${ }^{5}$ and Koji Takahashi, ${ }^{3,5}$ \\ ${ }^{1}$ College of Mechanical and Transportation Engineering, China University of Petroleum, Beijing 102249, China \\ ${ }^{2}$ Key Laboratory for Thermal Science and Power Engineering of Ministry of Education, Department of Engineering Mechanics, \\ Tsinghua University, Beijing 100084, China \\ ${ }^{3}$ International Institute for Carbon Neutral Energy Research (WPI-I2CNER), Kyushu University, Fukuoka 819-0395, Japan \\ ${ }^{4}$ Department of Mechanical Engineering, Kyushu University, Fukuoka 819-0395, Japan \\ ${ }^{5}$ Department of Aeronautics and Astronautics, Graduate School of Engineering, Kyushu University, Fukuoka 819-0395, Japan
}

Correspondence should be addressed to Xing Zhang; x-zhang@tsinghua.edu.cn

Received 19 February 2014; Revised 17 May 2014; Accepted 27 May 2014; Published 18 June 2014

Academic Editor: Theodorian Borca-Tasciuc

Copyright (c) 2014 Tingting Miao et al. This is an open access article distributed under the Creative Commons Attribution License, which permits unrestricted use, distribution, and reproduction in any medium, provided the original work is properly cited.

\begin{abstract}
Thin metal films are widely used as interconnecting wires and coatings in electronic devices and optical components. Reliable thermophysical properties of the films are required from the viewpoint of thermal management. The cross plane thermal transport of four polycrystalline molybdenum nanofilms with different thickness deposited on glass substrates has been studied by applying the picosecond laser transient thermoreflectance technique. The measurement is performed by applying both front pump-front probe and rear pump-front probe configurations with high quality signal. The determined cross plane thermal diffusivity of the Mo films greatly decreases compared to the corresponding bulk value and tends to increase as films become thicker, exhibiting significant size effect. The main mechanism responsible for the thermal diffusivity decrease of the present polycrystalline Mo nanofilms is the grain boundary scattering on the free electrons. Comparing the cross plane thermal diffusivity and inplane electrical conductivity indicates the anisotropy of the transport properties of the Mo films.
\end{abstract}

\section{Introduction}

As semiconductor/electronics technologies and micro-/ nanoelectromechanical systems (MEMS/NEMS) rapidly progress, thin metal films are widely used as interconnecting wires in the ultralarge-scale-integrated (ULSI) circuits $[1,2]$ and coatings [3] in many optical components and electronic devices. Due to film surface scattering and grain boundary scattering, the thermal conductivity of thin metal film drops significantly from its bulk value. As interconnecting wires, the thermophysical properties of metal nanofilms are crucial to ensure the capability and reliability of the delicate circuits systems. As coatings, the thermophysical properties of the metal nanofilms are required to deduce the dielectric films' thermal properties $[3,4]$.
Great efforts have been made on studying the cross plane thermal transport properties of thin metal films. Paddock and Eesley [5] first developed the picosecond thermoreflectance method and measured the cross plane thermal conductivities of Ni, Fe, Zr, and Ti films with different thickness. The determined cross plane thermal diffusivities exhibit significant size effect; that is, the thermal diffusivity of the $300 \mathrm{~nm}$ thick sputtered Ti film was 0.26 times the corresponding bulk value. Rohde [6] developed the photoacoustic method and measured the sputtered Au film and electroplated Ni film. The measured cross-plane thermal conductivity of the Au film $24 \mathrm{~W} \mathrm{~m}^{-1} \mathrm{~K}^{-1}$ is reduced by more than one order of magnitude compared with the bulk value of gold $314 \mathrm{~W} \mathrm{~m}^{-1} \mathrm{~K}^{-1}$, which is attributed to changes in the morphology of the film. Yamane et al. [7] developed the light-irradiation ac 


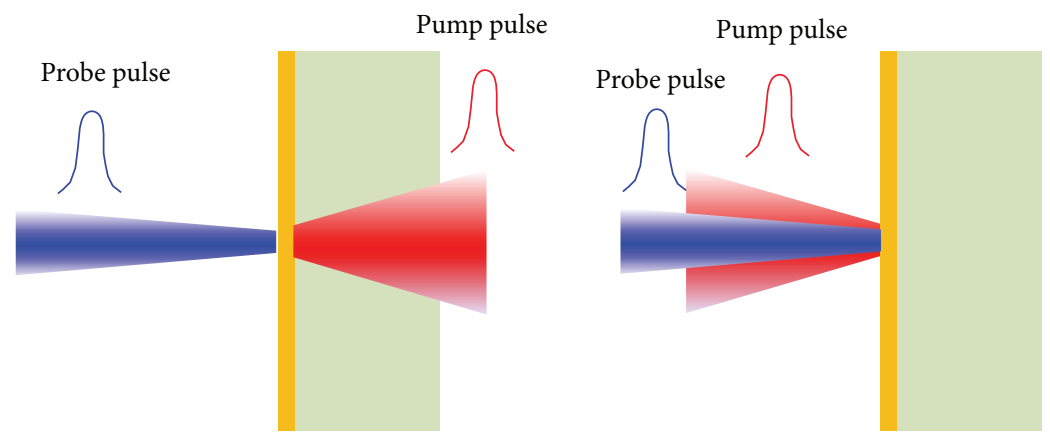

FiguRE 1: FF and RF configurations of the picosecond laser transient thermoreflectance technique.

calorimetric method and measured the cross plane thermal diffusivity of $\mathrm{Au}(30-340 \mathrm{~nm}), \mathrm{Ag}(30-230 \mathrm{~nm})$, and $\mathrm{Cu}(75-$ $1200 \mathrm{~nm}$ ) films. They found that the thermal diffusivity of the films decreases continuously as crystallite size falls below $60 \mathrm{~nm}$ and attributed the decrease to crystalline size and consequent restriction of the heat transfer of free electrons. Taketoshi et al. [8-13] have done series of works on developing the picosecond thermoreflectance system and studying the cross plane thermal transport in nanoscale metal films. Bourgoin et al. [14] studied the thermal conduction across thin metal films, including $\mathrm{Au}, \mathrm{Ag}, \mathrm{Sn}$, and $\mathrm{Al}$, by applying pump-probe laser measurements combined with numerical modeling based on the surface thermal lensing effect. Thermal conductivity of $\mathrm{Au}$ films was found to drop from 300 to $100 \mathrm{~W} \mathrm{~m}^{-1} \mathrm{~K}^{-1}$ when the film thickness is reduced from 2000 to $100 \mathrm{~nm}$. However, the measured results of the thin metal films scatter over a wide range. For instance, the determined cross plane thermal diffusivity of the Mo films with thickness about $100 \mathrm{~nm}$ ranges from $0.44 \times 10^{-5} \mathrm{~m}^{2} \mathrm{~s}^{-1}$ to $5.04 \times 10^{-5} \mathrm{~m}^{2} \mathrm{~s}^{-1}$ [8-13]. One mechanism responsible for the wide scatter is the synthesis condition, which has been illustrated by Taketoshi et al. [11].

In this paper, the cross plane thermal transport of four polycrystalline Mo nanofilms with thickness of $95.3 \mathrm{~nm}$, $200 \mathrm{~nm}, 400 \mathrm{~nm}$, and $600 \mathrm{~nm}$ deposited on glass substrates has been studied by applying the picosecond laser transient thermoreflectance technique, including front pump-front probe (FF) and rear pump-front probe (RF) configurations, at $300 \mathrm{~K}$. The determined cross plane thermal diffusivity of the films greatly decreases compared to the corresponding bulk value and tends to increase as films become thicker, exhibiting significant size effect.

\section{Experiment}

The picosecond laser transient thermoreflectance technique, including FF and RF configurations, is employed to study the cross plane thermal transport of polycrystalline Mo thin films, as shown in Figure 1. The picosecond pump pulses are applied to heat the front (or rear) surface of the studied film and thermal transport takes place across the film. For a metal, the surface temperature changes, resulting from pump pulse heating, will induce the reflectance alternation through the change in the complex dielectric function. The probe pulse follows the reflectance variation, that is, temperature, of the film front surface in real time. The cross plane thermal transport properties of the metal film can be extracted from fitting the time-resolved thermoreflectance signal with the theoretical predicted temperature profile.

According to the previous study [15], the time-dependent temperature profile of the thin metal film can be analyzed by applying mirror image method. When the pump pulse is applied to heat the front surface of the metal film in the FF mode, the time-dependent temperature response of the film front surface is [15]

$$
T_{\mathrm{FF}}(t)=\frac{1}{b_{f} \sqrt{\pi t}}\left(1+2 \sum_{n=1}^{\infty} \gamma^{n} \exp \left(-n^{2} \frac{\tau_{f}}{t}\right)\right),
$$

where $b_{f}$ is the thermal effusivity, defined as $b_{f}=\sqrt{\lambda_{f} c_{f} \rho_{f}}$, $\lambda_{f}$ is the thermal conductivity, $c_{f}$ is the specific heat capacity, and $\rho_{f}$ is the density of the thin metal film. $\tau_{f}$ is the heat diffusion time, and $\gamma$ is the multiplication coefficient for the amplitude of the virtual heat sources defined as [15]

$$
\gamma=\frac{b_{f}-b_{s}}{b_{f}+b_{s}}
$$

where $b_{s}$ is the thermal effusivity of the substrate.

When the pump pulse is applied to heat the rear surface of the metal film in the RF mode, the time-dependent temperature response of the front surface is [15]

$$
T_{\mathrm{RF}}(t)=\frac{2}{\left(b_{f}+b_{s}\right) \sqrt{\pi t}} \sum_{n=1}^{\infty} \gamma^{n} \exp \left(-\frac{(2 n+1)^{2} \tau_{f}}{4 t}\right) .
$$

From (1) and (3), it can be found that the heat diffusion time of the thin metal film can be determined by applying the FF or RF mode. Consequently, the cross plane thermal diffusivity of the thin metal film, $\alpha_{f}$, can be obtained through

$$
\alpha_{f}=\frac{d_{f}^{2}}{\tau_{f}}
$$

where $d_{f}$ is the thickness of the film.

The experimental setup, which is developed by PicoTherm Corporation, is schematically shown in Figure 2. 


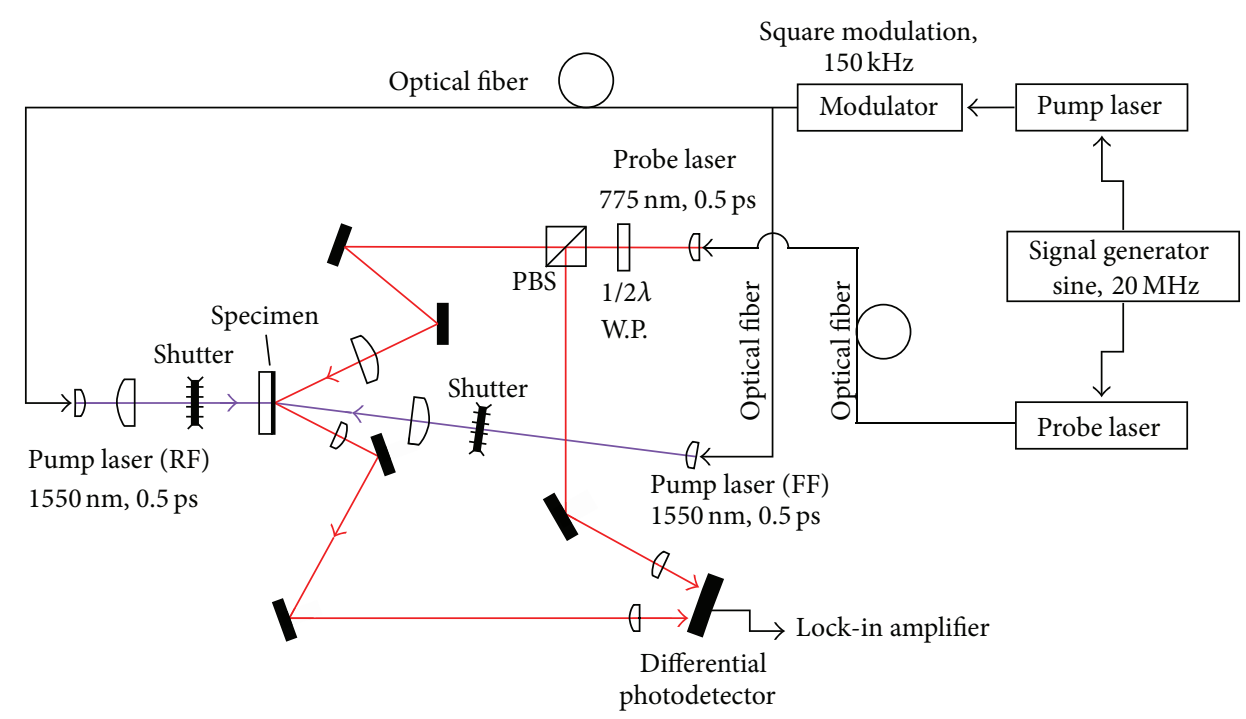

FIGURE 2: Schematic of the key elements of the picosecond laser transient thermoreflectance system.

The wavelengths of the pump and probe laser are $1550 \mathrm{~nm}$ and $775 \mathrm{~nm}$, respectively. The repetition rates of the pump and probe laser are tuned by the same signal generator of sine wave with frequency $20 \mathrm{MHz}$. The pulse width of the pump and probe pulse is broadened to about 0.5 ps upon propagation through optical fiber and lenses. The pump laser is divided into two beams for front and rear heating in FF and $\mathrm{RF}$ configurations by applying the optical fiber, and the probe laser is separated into two orthogonally polarized beams by a polarizing beam splitter (PBS) with the assistance of a half-wave plate for differential detection. The pump beam is modulated by an electrooptic modulator (EOM) with a frequency of $150 \mathrm{kHz}$ and then focused on the film with a spot diameter of $\sim 100 \mu \mathrm{m}$. The pump beam excites the sample and consequently creates a transient thermoreflectance occurring at the corresponding frequency. The probe beam is then focused on the heated area with a spot diameter of $\sim 50 \mu \mathrm{m}$ and used to monitor the reflectance change in real time. The delay time of the probe pulse with respect to the pump pulse is controlled by the signal generator. The probe beam reflected by the sample and the reference probe beam are detected by the differential photodetector with the assistance of a lock-in amplifier locked to the EOM frequency.

\section{Results and Discussion}

3.1. Effects of $\gamma$ and $\tau$. According to (1) and (3), the timeresolved front surface temperature change of the film is dominated by the thermal diffusion time $\tau$ and the multiplication coefficient $\gamma$, which are related to the thermal diffusivity and effusivity of the metal film and substrate. The effects of $\tau$ and $\gamma$ on the surface temperature are studied numerically. In the FF mode, the normalized time-resolved surface temperatures, for $\tau=0.6 \mathrm{~ns}$ and $\gamma=0.1,0.3,0.5,0.7$, and 0.9 , are compared in Figure 3(a), and the temperatures, for $\gamma=0.5$ and $\tau=0.2 \mathrm{~ns}, 0.4 \mathrm{~ns}, 0.6 \mathrm{~ns}, 0.8 \mathrm{~ns}$, and $1.0 \mathrm{~ns}$, are compared in Figure 3(b). It can be found that the multiplication coefficient $\gamma$ affects the initial temperature change weakly, that is, 0 $0.1 \mathrm{~ns}$, and significantly after $0.5 \mathrm{~ns}$, while the thermal diffusion time affects the temperature change significantly in the time range of $0-0.5 \mathrm{~ns}$ and weakly after $0.5 \mathrm{~ns}$. Hence, in the FF mode, from the experimentally obtained time-resolved temperature change, the cross plane thermal diffusion time and consequent thermal diffusivity of the metal film can be determined.

In the RF mode, the normalized time-resolved front surface temperatures, for $\tau=0.6 \mathrm{~ns}$ and $\gamma=0.1,0.3,0.5,0.7$, and 0.9, are compared in Figure 4(a), and the temperatures, for $\gamma=0.5$ and $\tau=0.2 \mathrm{~ns}, 0.4 \mathrm{~ns}, 0.6 \mathrm{~ns}, 0.8 \mathrm{~ns}$, and $1.0 \mathrm{~ns}$, are compared in Figure 4(b). It can be found that the multiplication coefficient $\gamma$ affects the initial temperature increase weakly while affecting the temperature decrease significantly. In contrast, the thermal diffusion time mainly affects the front surface temperature increase. Hence, in the $\mathrm{RF}$ mode, from the time-resolved temperature change, the thermal diffusion time and consequent thermal diffusivity of the metal film can be determined.

3.2. Thermal Diffusivity of Mo Films. The cross plane thermal diffusivities of four polycrystalline Mo nanofilms with thickness of $95.3 \mathrm{~nm}, 200 \mathrm{~nm}, 400 \mathrm{~nm}$, and $600 \mathrm{~nm}$ deposited on glass substrates have been studied by applying the picosecond laser transient thermoreflectance technique, including FF and RF modes, at $300 \mathrm{~K}$. The 200,400 , and $600 \mathrm{~nm}$ thick films have been grown by dc magnetron sputtering from rectangle $(381 \mathrm{~mm} \times 127 \mathrm{~mm}$ ) $99.99 \%$ Mo target in Ar low pressure of $0.4 \mathrm{~Pa}$ with flow rate $50 \mathrm{sccm}$. The chamber base pressure is $10^{-3} \mathrm{~Pa}$, the sputtering power is $2400 \mathrm{~W}$, and the substrate temperature is $250^{\circ} \mathrm{C}$. The $95.3 \mathrm{~nm}$ thick film is deposited by dc magnetron sputtering from circular $(38.1 \mathrm{~mm}$ in diameter) $99.95 \%$ Mo target in Ar low pressure of $1 \mathrm{~Pa}$ with flow rate $5 \mathrm{sccm}$. The chamber base pressure is $7 \times 10^{-4} \mathrm{~Pa}$, the sputtering power is $100 \mathrm{~W}$, and the substrate is not heated. In the FF mode, the time-dependent thermoreflectance signals 


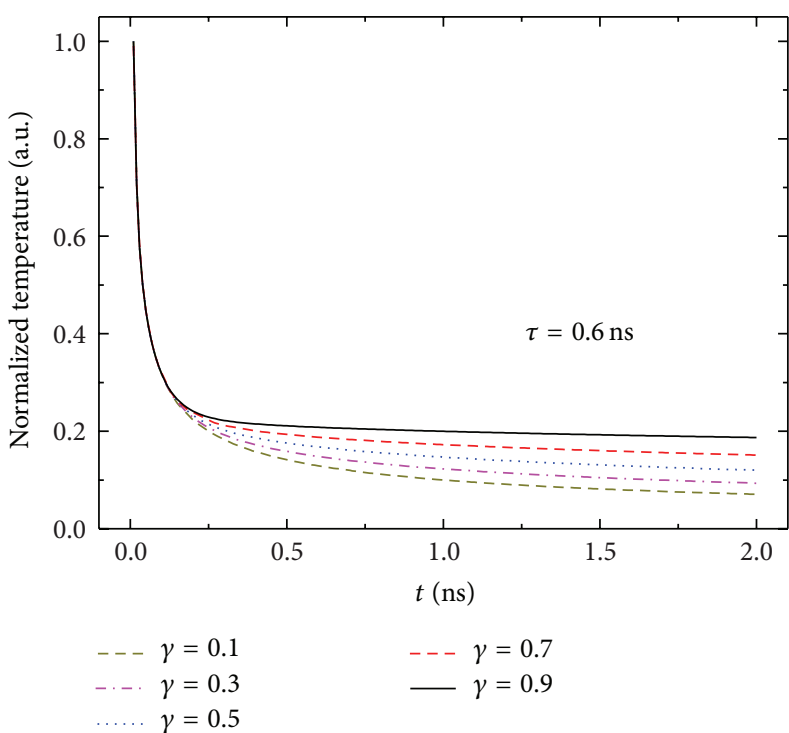

(a)

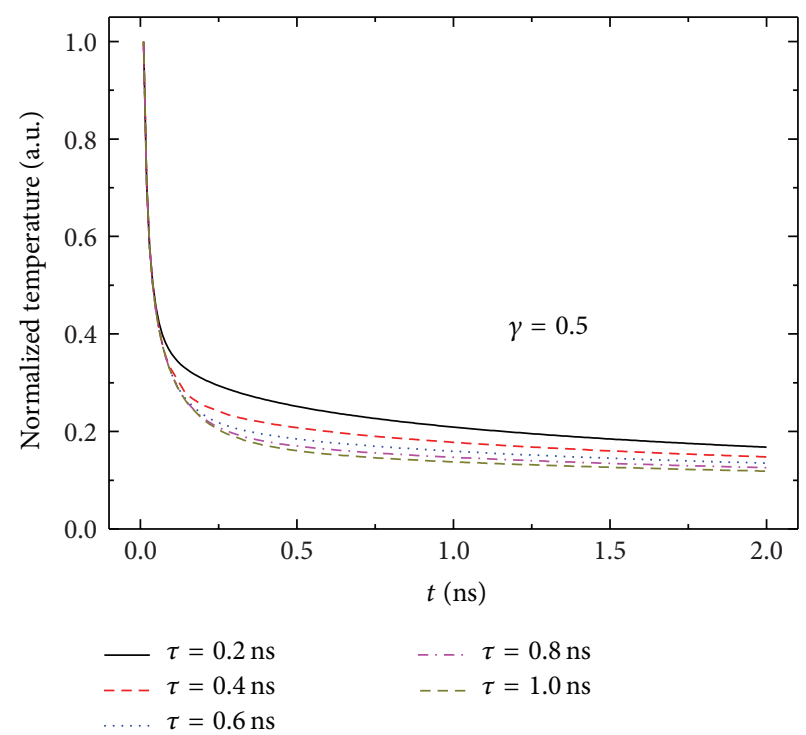

(b)

FIGURE 3: Normalized time-resolved surface temperature of the metal film in FF configuration: (a) for different $\gamma$ and (b) for different $\tau$.

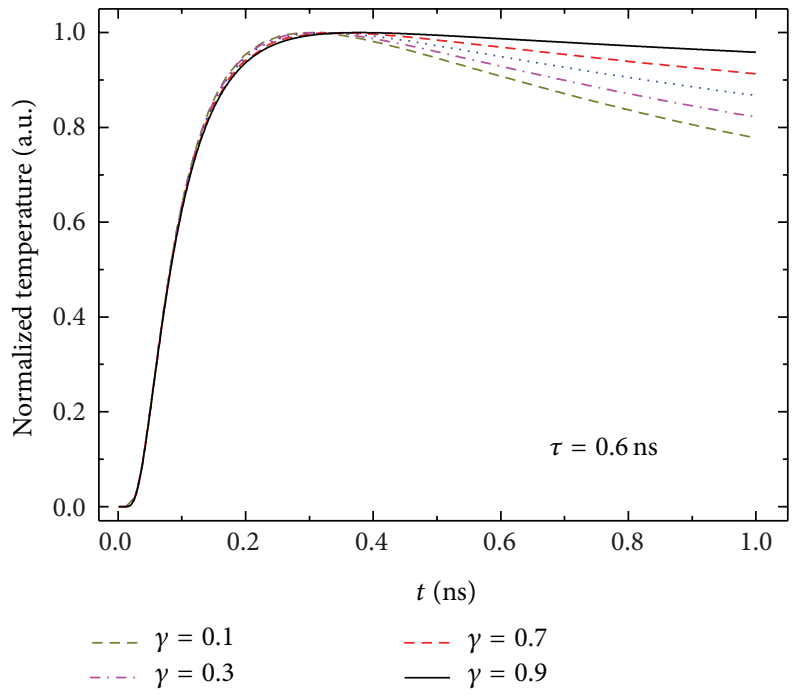

(a)

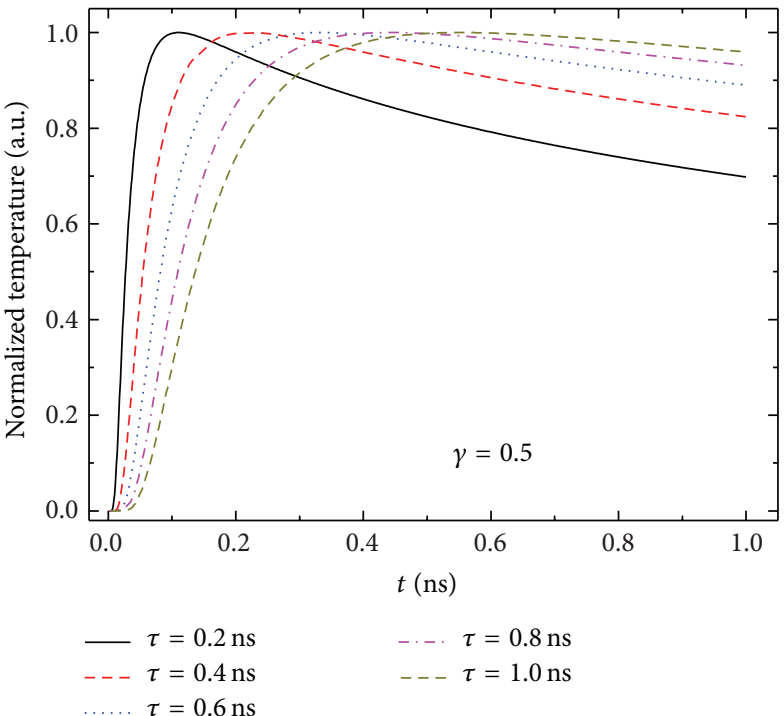

(b)

FIGURE 4: Normalized time-resolved surface temperature of the metal film in RF configuration: (a) for different $\gamma$ and (b) for different $\tau$.

of the Mo films with different thickness are compared in Figure 5. It can be found that the signal is of high quality with high signal-to-noise ratio. Qualitatively, by comparing the temperature history curve of the films with Figure 3(b), it can be found that the thermal diffusion time increases as films become thicker. Quantitatively, the transient temperature curve is fitted based on (1) with heat diffusion time $\tau$ and multiplication coefficient $\gamma$ as fitting parameters. As illustrated in Figure 5, the theoretical fittings agree well with the experimental data in the whole delay time range. The determined thermal diffusion times of the 95.3, 200, and $400 \mathrm{~nm}$ thick Mo films are $0.430 \mathrm{~ns}, 1.10 \mathrm{~ns}$, and $3.66 \mathrm{~ns}$, respectively. Accordingly, the thermal diffusivities of the 95.3, 200 , and $400 \mathrm{~nm}$ thick Mo films extracted from (4) are $2.11 \times 10^{-5} \mathrm{~m}^{2} \mathrm{~s}^{-1}, 3.63 \times 10^{-5} \mathrm{~m}^{2} \mathrm{~s}^{-1}$, and $4.38 \times 10^{-5} \mathrm{~m}^{2} \mathrm{~s}^{-1}$, respectively, while the thermal diffusivity of bulk Mo is $5.4 \times$ $10^{-5} \mathrm{~m}^{2} \mathrm{~s}^{-1}$. It can be found that, in the present Mo films, the cross plane thermal diffusivity greatly decreases compared to the bulk value and tends to increase as films become thicker, exhibiting significant size effect.

To verify the measurement result of FF mode, the thin Mo films are also studied by applying RF mode. First, we study the $95.3 \mathrm{~nm}$ thick film, plot the best-fit curve along with the experimental data, and obtain the thermal diffusivity, 


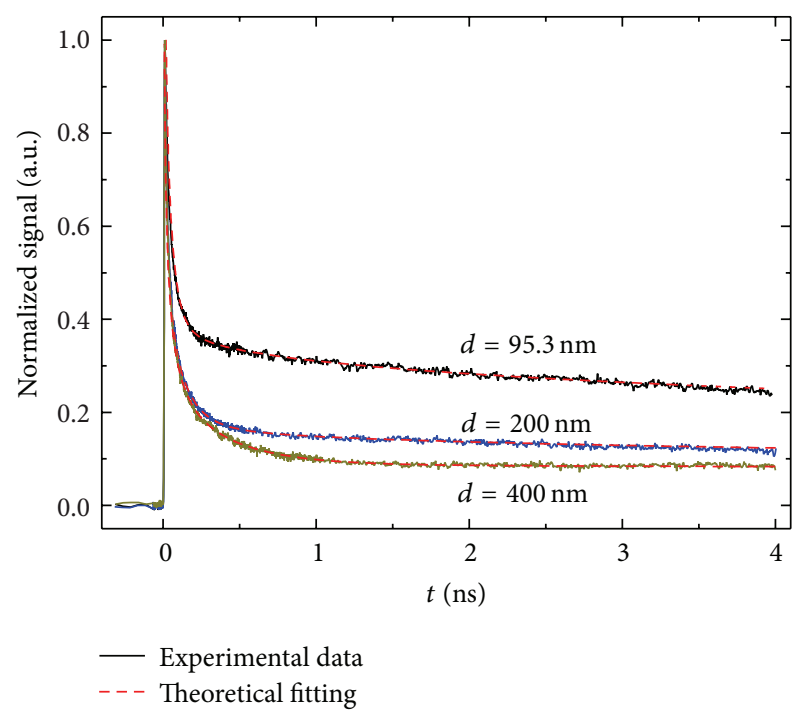

FIGURE 5: Time-dependent thermoreflectance signal of the Mo films with different thickness measured in the FF mode.

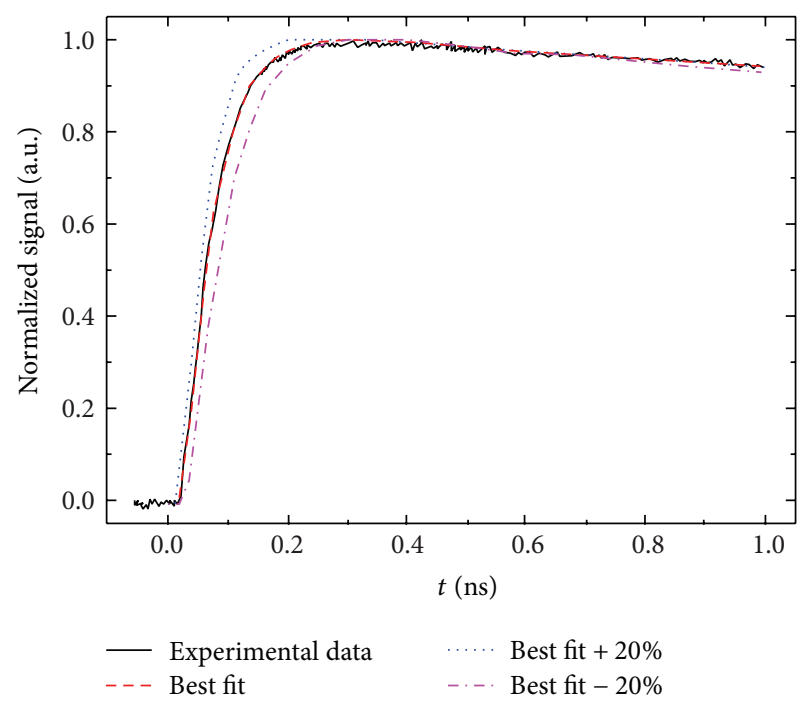

FIGURE 6: Experiment data and best-fit curves for thermal diffusivity of $95.3 \mathrm{~nm}$ thick Mo film and solutions obtained by varying thermal diffusivity by $20 \%$ are also shown.

$2.00 \times 10^{-5} \mathrm{~m}^{2} \mathrm{~s}^{-1}$. Then vary thermal diffusivity by $20 \%$ to see how the theoretical predicted thermoreflectance changes. Figure 6 shows the three sets of predicted time-resolved thermoreflectance with different thermal diffusivity and gives a visual picture of the sensitivity to the thermal diffusivity of the metal films. It can be observed that the present measurement is sensitive enough to detect the thermal diffusivity of the thin Mo films.

The normalized time-resolved thermoreflectance responses of the films with different thickness are compared in Figure 7. It can be found clearly that the temperature rises slower as films become thicker. The thermal diffusion time of the $600 \mathrm{~nm}$ thick film is the longest, while the $95.3 \mathrm{~nm}$ thick film is the shortest. The temperature history curve is fitted

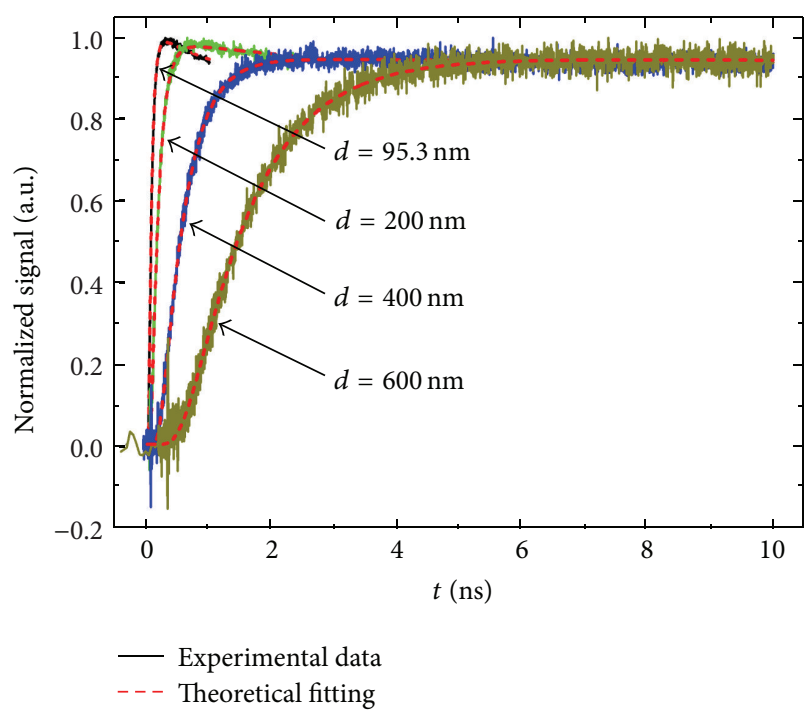

FIgURE 7: Time-dependent thermoreflectance signal of the Mo films with different thickness measured in the RF mode.

based on (3) with heat diffusion time $\tau$ and multiplication coefficient $\gamma$ as fitting parameters. As illustrated in Figure 7, the theoretical fittings agree well with the experimental data in the whole delay time range. The determined thermal diffusion times of the 95.3, 200, 400, and $600 \mathrm{~nm}$ thick Mo films are $0.453 \mathrm{~ns}, 1.13 \mathrm{~ns}, 3.66 \mathrm{~ns}$, and $9.99 \mathrm{~ns}$, respectively. Accordingly, the extracted thermal diffusivities of the 95.3, 200,400 , and $600 \mathrm{~nm}$ thick films are $2.00 \times 10^{-5} \mathrm{~m}^{2} \mathrm{~s}^{-1}$, $3.53 \times 10^{-5} \mathrm{~m}^{2} \mathrm{~s}^{-1}, 4.31 \times 10^{-5} \mathrm{~m}^{2} \mathrm{~s}^{-1}$, and $3.60 \times 10^{-5} \mathrm{~m}^{2} \mathrm{~s}^{-1}$.

The measurement results of the FF and RF modes match well with each other, indicating that the determined thermal diffusivity of thin Mo films from picosecond laser transient thermoreflectance technique is convincing. The determined cross plane thermal diffusivity of the present Mo films greatly decreases compared to the corresponding bulk value and tends to increase as films become thicker, exhibiting significant size effect.

The thermal diffusivity of Mo films with different thickness, including present Mo films and previous reported samples [9-13], is summarized in Figure 8. It can be found that the thermal diffusivity of most of the Mo films decreases compared to the corresponding bulk value, except for a few reported values.

The thin Mo films are polycrystalline and the mean grain size is comparable to or less than the bulk electron mean free path. The grain boundary scattering on free electrons degrades the thermal transport. In addition, the free electrons will also be scattered by film surface and impurities. Because of high purity of the Mo film, the contribution made by the impurity scattering can be neglected [16]. Moreover, the effect of surface scattering on the cross plane thermal transport is sufficiently weak compared to grain boundary scattering, since the film surface is boundary for cross plane thermal transport. To evaluate the effect of grain boundary scattering on thermal transport, Qiu and Tien [17] combined the electrical-thermal transport analogy, Wiedemann-Franz 

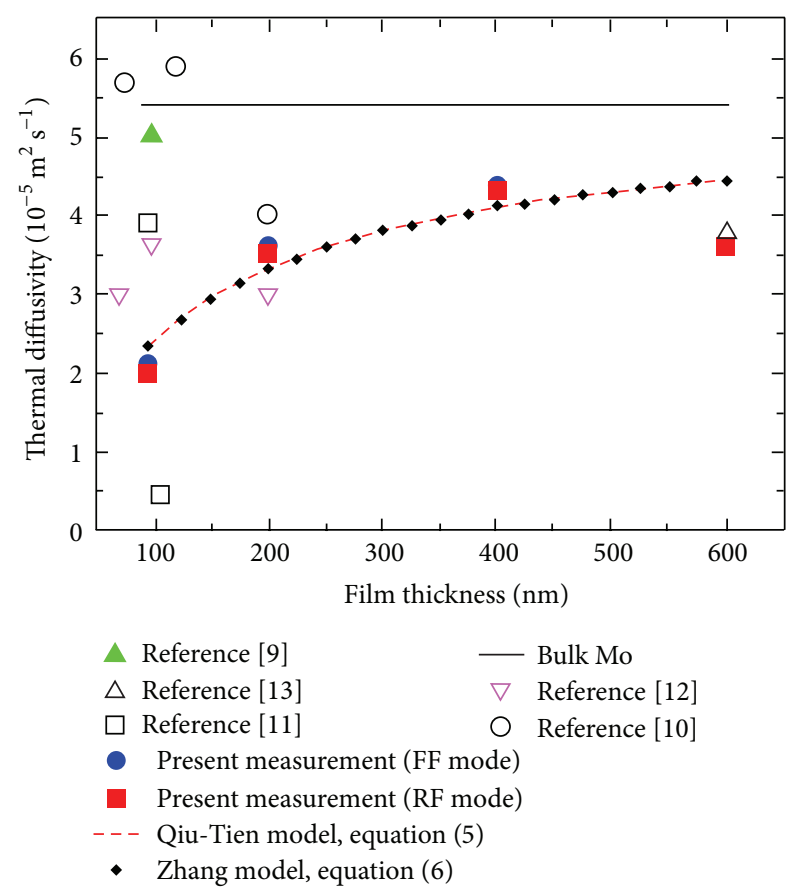

Figure 8: Summarized thermal diffusivity of the Mo films with different thickness.

law, and Mayadas-Shatzkes theory [18, 19], which gives the grain boundary scattering effect on the electrical transport of polycrystalline metal films by assuming that the grain boundary can be represented by a $\delta$-function potential, and obtained the thermal conductivity of polycrystalline metal films as follows:

$$
\begin{array}{r}
\frac{\lambda_{f}}{\lambda_{b}}=1-\frac{3}{2} \alpha+3 \alpha^{2}-3 \alpha^{3} \ln \left(1+\frac{1}{\alpha}\right), \\
\alpha=\frac{l_{b}}{d} \frac{R}{1-R},
\end{array}
$$

where $\lambda_{f}$ and $\lambda_{b}$ are the thermal conductivity of polycrystalline film and the corresponding bulk value, $l_{b}$ is the bulk electron mean free path, and $R$ denotes the grain boundary reflection coefficient of conduction electrons. Zhang et al. [20-22] considered the electron effective relaxation time determined by both background scattering and grain boundary scattering based on Matthiessen's rule and obtained the thermal conductivity of the polycrystalline metal film as follows:

$$
\frac{\lambda_{f}}{\lambda_{b}}=\frac{1}{1+R l_{b} / d} .
$$

Previous measurement [13] showed that the thermal diffusivity of the $600 \mathrm{~nm}$ thick Mo films prepared by a dc sputtering technique with average grain size $27 \mathrm{~nm}$ is $3.8 \times$ $10^{-5} \mathrm{~m}^{2} \mathrm{~s}^{-1}$. From (5), the extracted grain boundary reflection coefficient of the polycrystalline Mo films, $R$, is about 0.35 , and, according to (6), the extracted grain boundary reflection coefficient is 0.78 . Previous studies [23-26] have illustrated

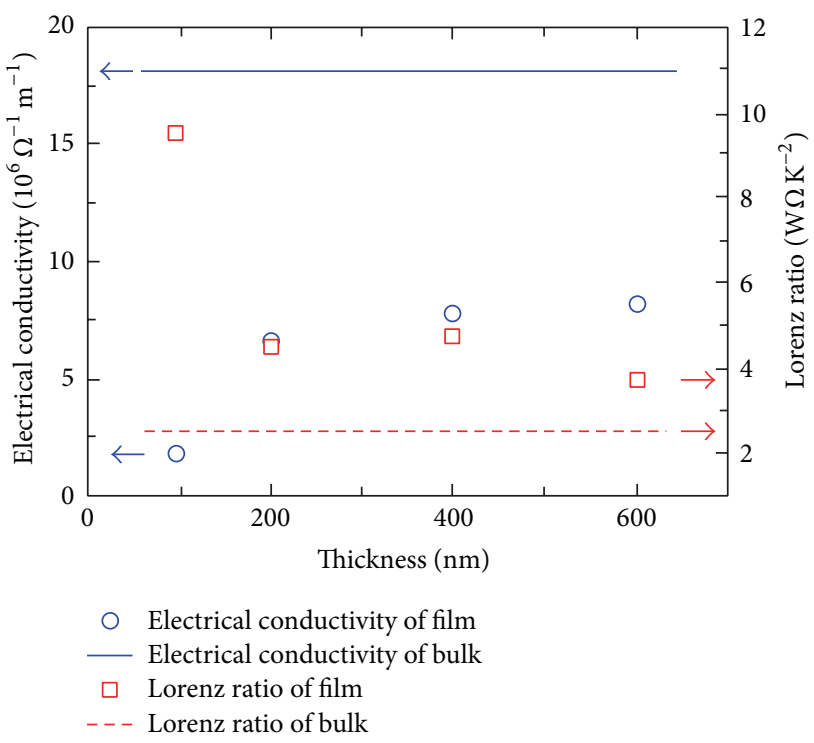

FIGURE 9: Electrical conductivity and Lorenz ratio of the Mo films with different thickness.

that, in thin metal films, the grain size is usually proportional to the film thickness above a thickness of $10 \mathrm{~nm}$. The predicted thickness-dependent thermal diffusivities of the thin Mo films of different thickness according to (5) and (6) are illustrated as dash line and scattered diamonds in Figure 8. It can be found that the grain boundary scattering decreases the cross plane thermal diffusivity of Mo films greatly and the effect weakens as films become thicker. The theoretical predictions from (5) and (6) match well with each other and agree with the experimental data in the range of $95.3 \mathrm{~nm}$ to $400 \mathrm{~nm}$. The thermal diffusivity of the $600 \mathrm{~nm}$ thick film is smaller than that of $400 \mathrm{~nm}$ thick film, and the possible mechanism is that the grain size of the $600 \mathrm{~nm}$ thick film is smaller.

The inplane electrical conductivity of the Mo films is measured by using a Loresta GP resistivity meter (MCP-T610 model, Mitsubishi Chemical Co., Japan). According to the Wiedemann-Franz law, for metals at not too low temperatures, the ratio of the thermal conductivity to the electrical conductivity is directly proportional to the temperature and can be expressed in terms of the ratio as follows:

$$
\frac{\lambda}{\sigma T}=L
$$

where $L$ is the Lorenz ratio and the value is $\pi^{2} k_{B}^{2} /\left(3 e^{2}\right)=$ $2.45 \times 10^{-8} \mathrm{~W} \Omega \mathrm{K}^{-2}$. The determined electrical conductivity and Lorenz ratio of the films as well as the corresponding bulk value are illustrated in Figure 9. It can be found that the inplane electrical conductivity of the films greatly decreases compared to that of bulk Mo, exhibiting significant size effect. The Lorenz ratio of the films is larger than the bulk value. The present Lorenz ratio is determined from the cross plane thermal conductivity and inplane electrical conductivity. Hence, the larger Lorenz ratio indicates the anisotropy of the transport properties of the present Mo films. There are two 
possible mechanisms responsible for the anisotropy. One is that the anisotropy of the nanostructure of the sputtered film, which is composed of thin columnar grains perpendicular to the film surface $[11,13]$, and the other is that the inplane transport needs to pass through much more grain boundaries than the cross plane transport.

\section{Conclusion}

The cross plane thermal diffusivity of four polycrystalline Mo nanofilms in the thickness range of 95.3-600 nm deposited on glass substrates has been measured by applying the picosecond laser transient thermoreflectance technique at 300 K. Numerical simulation and experimental measurement illustrate that both FF and RF modes can be used to determine the thermal diffusivity of metal films and the measurement result is convincing with high signal-to-noise ratio. The determined cross plane thermal diffusivity of the Mo films greatly decreases compared to the corresponding bulk value and tends to increase as films become thicker, exhibiting significant size effect. The grain boundary scattering on the free electrons is mainly responsible for the thermal diffusivity decrease. The thermal diffusivity of the $600 \mathrm{~nm}$ thick film is smaller than that of $400 \mathrm{~nm}$ thick film, and the possible mechanism is that the grain size of the $600 \mathrm{~nm}$ thick film is smaller. Comparing the cross plane thermal diffusivity and inplane electrical conductivity indicates the anisotropy of the transport properties of the present Mo films.

\section{Conflict of Interests}

The authors declare that there is no conflict of interests regarding the publication of this paper.

\section{Acknowledgments}

This work was supported by the National Natural Science Foundation of China (Grant nos. 51206094 and 51327001) and Science Foundation of China University of Petroleum, Beijing (2462013YJRC027).

\section{References}

[1] S. P. Murarka, "Multilevel interconnections for ULSI and GSI era," Materials Science and Engineering R: Reports, vol. 19, no. 3-4, pp. 87-151, 1997.

[2] H. G. Craighead, "Nanoelectromechanical systems," Science, vol. 290, no. 5496, pp. 1532-1535, 2000.

[3] G. Chen and P. Hui, "Thermal conductivities of evaporated gold films on silicon and glass," Applied Physics Letters, vol. 74, no. 20, pp. 2942-2944, 1999.

[4] G. Chen and P. Hui, "Pulsed photothermal modeling of composite samples based on transmission-line theory of heat conduction," Thin Solid Films, vol. 339, no. 1-2, pp. 58-67, 1999.

[5] C. A. Paddock and G. L. Eesley, "Transient thermoreflectance from thin metal films," Journal of Applied Physics, vol. 60, no. 1, pp. 285-290, 1986.
[6] M. Rohde, "Photoacoustic characterization of thermal transport properties in thin films and microstructures," Thin Solid Films, vol. 238, no. 2, pp. 199-206, 1994.

[7] T. Yamane, Y. Mori, S.-I. Katayama, and M. Todoki, "Measurement of thermal diffusivities of thin metallic films using the ac calorimetric method," Journal of Applied Physics, vol. 82, no. 3, pp. 1153-1156, 1997.

[8] N. Taketoshi, T. Baba, and A. Ono, "Development of a thermal diffusivity measurement system for metal thin films using a picosecond thermoreflectance technique," Measurement Science and Technology, vol. 12, no. 12, pp. 2064-2073, 2001.

[9] N. Taketoshi, T. Baba, and A. Ono, "Observation of heat diffusion across submicrometer metal thin films using a picosecond thermoreflectance technique," Japanese Journal of Applied Physics 2: Letters A, vol. 38, no. 11, pp. L1268-L1271, 1999.

[10] N. Taketoshi, T. Baba, E. Schaub, and A. Ono, "Homodyne detection technique using spontaneously generated reference signal in picosecond thermoreflectance measurements," Review of Scientific Instruments, vol. 74, no. 12, pp. 5226-5230, 2003.

[11] N. Taketoshi, T. Yagi, and T. Baba, "Effect of synthesis condition on thermal diffusivity of molybdenum thin films observed by a picosecond light pulse thermoreflectance method," Japanese Journal of Applied Physics, vol. 48, no. 5, Article ID EC014, 2009.

[12] T. Baba, N. Taketoshi, and T. Yagi, "Development of ultrafast laser flash methods for measuring thermophysical properties of thin films and boundary thermal resistances," Japanese Journal of Applied Physics, vol. 50, no. 11, Article ID 11RA01, 2011.

[13] S. H. Firoz, T. Yagi, N. Taketoshi, K. Ishikawa, and T. Baba, "Direct observation of thermal energy transfer across the thin metal film on silicon substrates by a rear heating-front detection thermoreflectance technique," Measurement Science and Technology, vol. 22, no. 2, Article ID 024012, 2011.

[14] J.-P. Bourgoin, G.-G. Allogho, and A. Haché, “Thermal conduction in thin films measured by optical surface thermal lensing," Journal of Applied Physics, vol. 108, no. 7, Article ID 073520, 2010.

[15] T. Baba, "Analysis of one-dimensional heat diffusion after light pulse heating by the response function method," Japanese Journal of Applied Physics, vol. 48, no. 5, Article ID EB041, 2009.

[16] P. G. Klemens and R. K. Williams, "Thermal conductivity of metals and alloys," International Metals Reviews, vol. 31, no. 5, pp. 197-215, 1986.

[17] T. Q. Qiu and C. L. Tien, "Size effects on nonequilibrium laserheating of metal-films," Journal of Heat Transfer-Transactions of the ASME, vol. 115, no. 4, pp. 842-847, 1993.

[18] A. F. Mayadas, M. Shatzkes, and J. F. Janak, "Electrical resistivity model for polycrystalline films: the case of specular reflection at external surfaces," Applied Physics Letters, vol. 14, no. 11, pp. 345-347, 1969.

[19] A. F. Mayadas and M. Shatzkes, "Electrical-resistivity model for polycrystalline films: the case of arbitrary reflection at external surfaces," Physical Review B, vol. 1, no. 4, pp. 1382-1389, 1970.

[20] X. Zhang, K. Takahashi, and M. Fujii, "Charge and heat transport in polycrystalline metallic nanostructures," Chinese Physics Letters, vol. 25, no. 9, pp. 3360-3363, 2008.

[21] W.-G. Ma, H.-D. Wang, X. Zhang, and K. Takahashi, "Different effects of grain boundary scattering on charge and heat transport in polycrystalline platinum and gold nanofilms," Chinese Physics B, vol. 18, no. 5, pp. 2035-2040, 2009.

[22] H.-D. Wang, J.-H. Liu, X. Zhang, Z.-Y. Guo, and K. Takahashi, "Experimental study on the influences of grain boundary scattering on the charge and heat transport in gold and platinum 
nanofilms," Heat and Mass Transfer/Waerme- und Stoffuebertragung, vol. 47, no. 8, pp. 893-898, 2011.

[23] J. W. C. De Vries, “Temperature and thickness dependence of the resistivity of thin polycrystalline aluminium, cobalt, nickel, palladium, silver and gold films," Thin Solid Films, vol. 167, no. 1-2, pp. 25-32, 1988.

[24] C. Charton and M. Fahland, "Growth of Ag films on PET deposited by magnetron sputtering," Vacuum, vol. 68, no. 1, pp. 65-73, 2002.

[25] C. Charton and M. Fahland, "Electrical properties of Ag films on polyethylene terephthalate deposited by magnetron sputtering," Thin Solid Films, vol. 449, no. 1-2, pp. 100-104, 2004.

[26] J. S. Jin, J. S. Lee, and O. Kwon, "Electron effective mean free path and thermal conductivity predictions of metallic thin films," Applied Physics Letters, vol. 92, no. 17, Article ID 171910, 2008. 

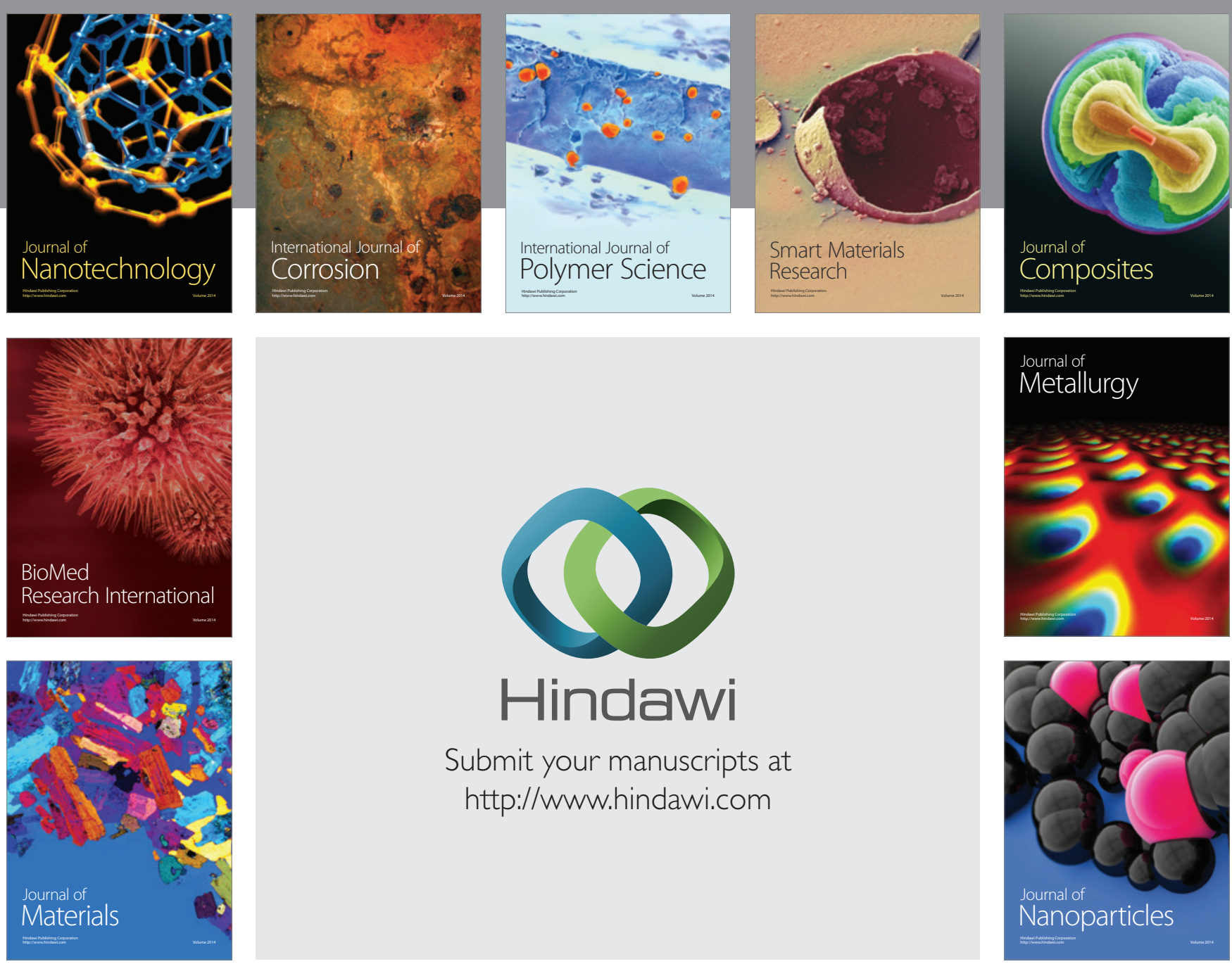

Submit your manuscripts at http://www.hindawi.com
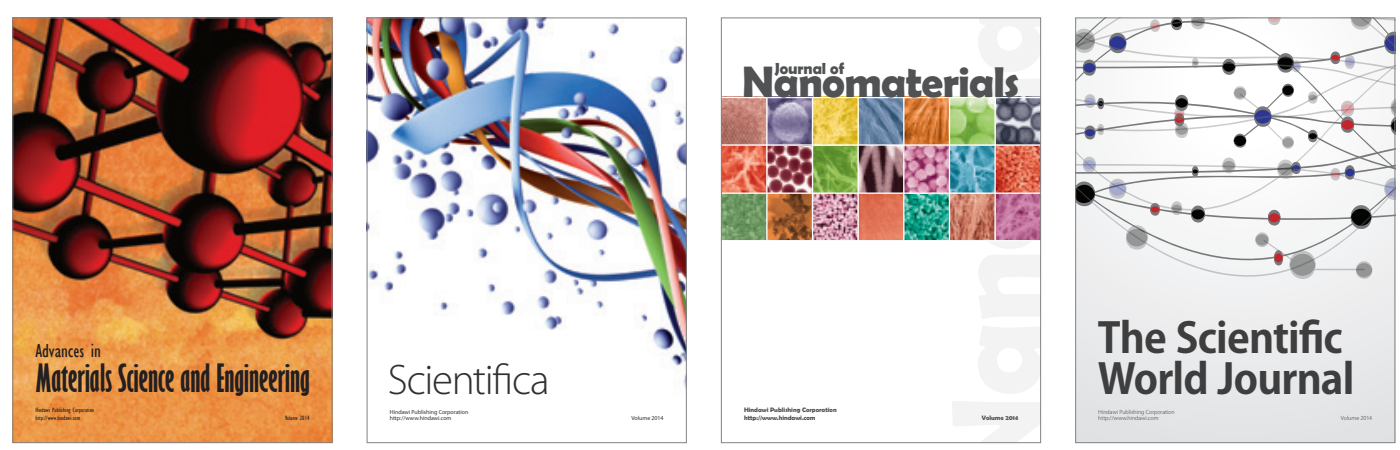

\section{The Scientific World Journal}
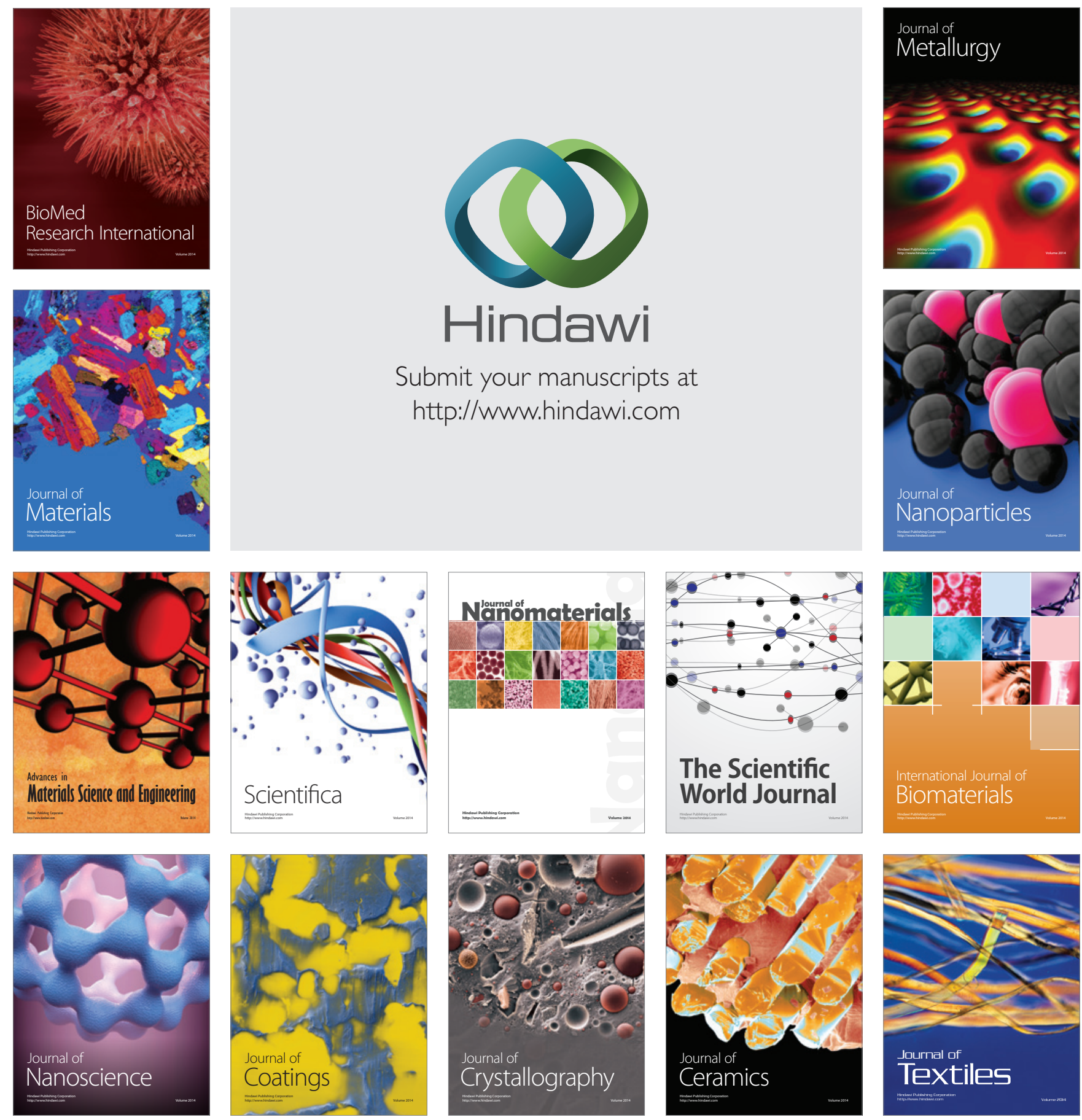